\title{
Session VIII
}

\section{Circumstellar Shells}




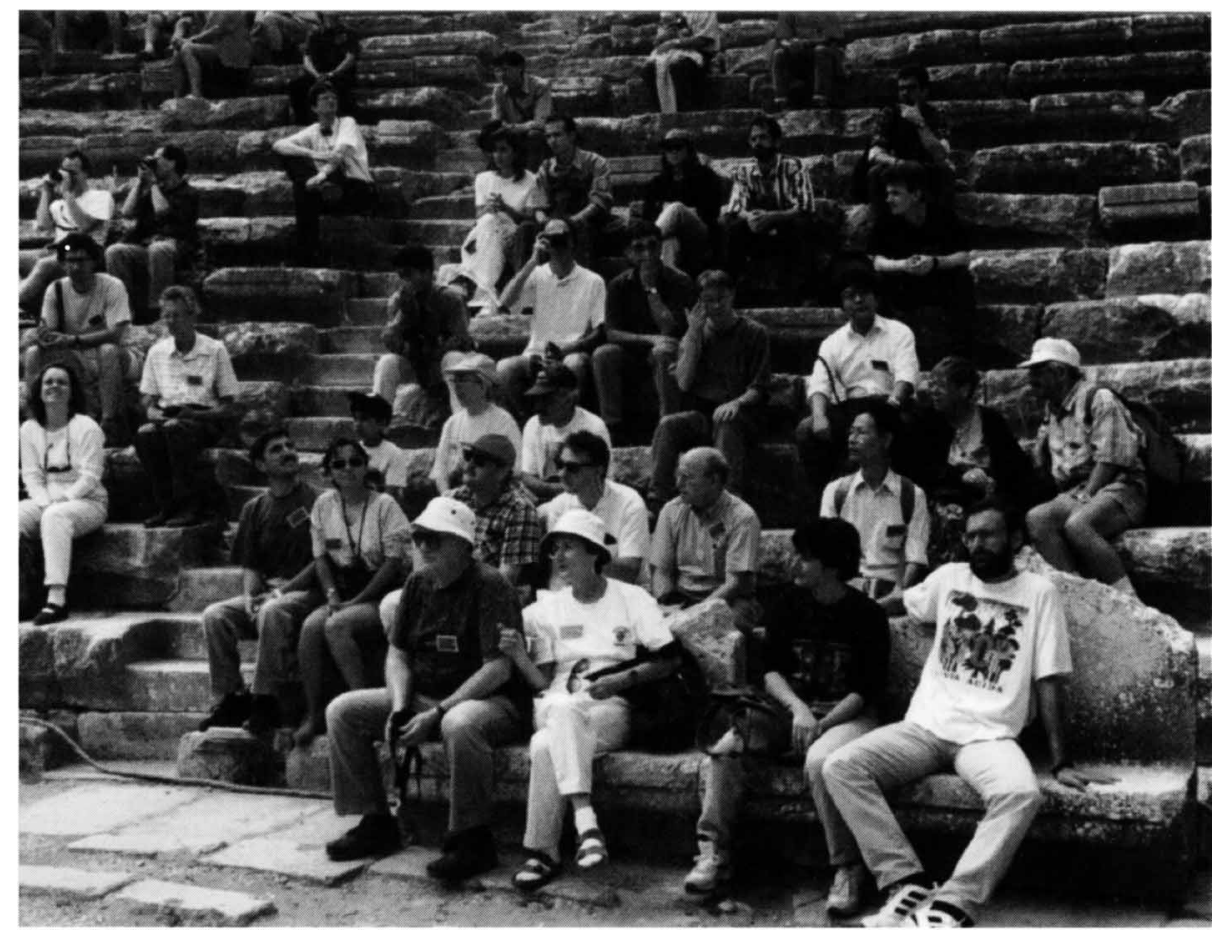

Participants reclining on the 1850-year-old stone seats of the theater at Aspendos, watching nothing in particular. 


\title{
CIRCUMSTELLAR DUST AROUND M, S AND C STARS
}

\author{
IRENE R. LITTLE-MARENIN \\ Wellesley College \\ Wellesley, MA 02181, U.S.A.
}

\begin{abstract}
The circumstellar shells of $\mathrm{M}$ stars produce emission features due to amorphous silicates peaking around 10 and $18 \mu \mathrm{m}$, with additional emission at $11 \mu \mathrm{m}$ due to crystalline olivine and at $13.1 \mu \mathrm{m}$ (unknown carrier). $\mathrm{C}$ stars are associated with $\mathrm{SiC}$ dust emission at $11.2 \mu \mathrm{m}$ with additional emission around $8.9 \mu \mathrm{m}$, whereas $\mathrm{S}$ stars have a relatively weak $10.5 \mu \mathrm{m}$ emission feature which is due neither to silicates nor to SiC.
\end{abstract}

\section{Introduction}

With the advent of IRAS a new episode of infrared astronomy began with the identification of hundreds of thousands of infrared sources. Thousands of these sources were bright enough ( $>2$ Jansky) to have their spectra recorded in the 8-22 $\mu \mathrm{m}$ region with the Low Resolution Spectrometer (LRS). This wavelength region is rich with different types of dust emission features. The existence of dust in circumstellar envelopes has been known for many decades. Stars on the AGB eject copious quantities of gas into space, part of which will condense into solid grains after the gas has cooled to $\mathrm{T}<1500 \mathrm{~K}$. The type of grain that is produced depends on the composition of the out-flowing material. In the oxygen-rich shells $(\mathrm{C} / \mathrm{O}<1)$ that are associated with $\mathrm{M}$ stars, the oxygen remaining after the formation of $\mathrm{CO}$ will condense into oxygen-rich solids such as amorphous silicates with emission features around 10 and $18 \mu \mathrm{m}$. On the other hand, in the carbon-rich shells $(\mathrm{C} / \mathrm{O}>1)$ associated with $\mathrm{C}$ stars, the extra carbon will condense into $\mathrm{SiC}$ grains with an emission feature around $11.2 \mu \mathrm{m}$.

IRAS was very good in characterizing large numbers of LRS by their major emission and absorption features. However, noisy spectra at times were assigned incorrect characterizations and previously unknown features could not be accommodated by the classification scheme. 


\section{The M stars}

In 1990, Little-Marenin and Little (LML90) analyzed IRAS LRS spectra of Mira variables and showed that the $10 \mu \mathrm{m}$ silicate emission feature (called class Sil) showed additional dust components at $11 \mu \mathrm{m}$ (crystalline olivine; class Sil+) and at $13.1 \mu \mathrm{m}$ (class 3C). The carrier of the $13.1 \mu \mathrm{m}$ feature has not yet been unambiguously identified, but it does not appear to match the characteristics of corundum (Sloan et al. 1996). The strength of the 11 $\mu \mathrm{m}$ feature at times rivaled or surpassed the strength of the $10 \mu \mathrm{m}$ feature (class Sil++). A weak, broad feature extending from 9 to $15 \mu \mathrm{m}$ was also identified, possibly due to aluminum oxide. Besides the $10 \mu \mathrm{m}$ feature, amorphous silicate has a long-wavelength feature that peaks at $18 \mu \mathrm{m}$. In stars which show Sil+ and 3C features, we found that the long-wavelength feature peaks at $19 \mu \mathrm{m}$ rather than at $18 \mu \mathrm{m}$.

Two recent studies (Hron et al. 1997; Sloan \& Price 1995) have done much to quantify the characteristics of the different types of dust emission seen in $M$ stars. The two methods emphasize different aspects of the emission. Sloan and Price wanted to investigate correlations between the total amount of dust emission and the shape of the emission longward of $10 \mu \mathrm{m}$ by using the flux ratios $F_{10} / F_{11}$ and $F_{11} / F_{12}$. They showed that the total dust emission in $\mathrm{M}$ stars is constrained to a narrow sequence of shapes ranging from the classic narrow feature peaking around $10 \mu \mathrm{m}$ to the broad, low-contrast feature which peaks between 11 and $12 \mu \mathrm{m}$. They use this sequence to classify emission features of AGB stars. The LML90 classes more or less fall in different segments of their distribution. A limitation of their method is that they only used the longward portion of the emission features, whereas there are clear differences in the shape of the various classes that can be identified shortward of $10 \mu \mathrm{m}$.

Hron et al. on the other hand concentrated on investigating the shapes of the emission features by first subtracting a photospheric and dust "continuum" from the LRS. Using ratios of the remaining integrated flux in both the short- and long-wavelength portion of the feature, they found that they could recover the various LML90 classes. Their analysis showed that Miras on average tend to have thicker shells and stronger features than semiregular variables (SRb). It is unclear if their conclusion that Miras have lower stellar temperatures is not an artifact of their method. The $13.1 \mu \mathrm{m}$ component appears to be associated primarily with SRb variables and with a fairly narrow range of shell optical depth. Sloan et al. (1996) also found that the $13.1 \mu \mathrm{m}$ feature is associated primarily with SRb variables and occurs in approximately $75-90 \%$ of these sources.

At large optical depths in the shell, the silicate features go into absorption. It is easy to confuse a partially self-absorbed silicate feature seen in 
M stars with the $\mathrm{SiC}$ plus 8-9 $\mu \mathrm{m}$ feature seen in $\mathrm{C}$ stars, as has been done in several papers in the literature.

A few AGB stars show no dust emission features.

\section{The C Stars}

The circumstellar shells of many $\mathrm{C}$ stars are characterized by $\mathrm{SiC}$ dust emission at $11.2 \mu \mathrm{m}$. Unlike the $10 \mu \mathrm{m}$ silicate feature, we found the $\mathrm{SiC}$ feature to be so uniform that it could be used for classification purposes (Little-Marenin et al. 1987). In a few stars (Y CVn, RY Dra, IRC+10216) the $\mathrm{SiC}$ feature is shifted so that it peaks at $11.4 \mu \mathrm{m}$.

We (Little-Marenin et al. 2000) and Goebel et al. (1995) have identified an emission feature in the $8-9 \mu \mathrm{m}$ region possibly due to $a: \mathrm{C}-\mathrm{H}$, as well as absorption in the $13-15 \mu \mathrm{m}$ region due to $\mathrm{C}_{2} \mathrm{H}_{2}$ and $\mathrm{HCN}$. A few stars show a weak, broad emission feature which is clearly different in shape from the aluminum oxide feature seen in M stars. Its carrier has not as yet been identified.

After removing an estimated stellar contribution, we find that the majority of our sources fall into two categories: spectra with the classic $\mathrm{SiC}$ emission feature peaking around $11.2-11.5 \mu \mathrm{m}$ (we will call this class $\mathrm{SiC}$ ), and spectra where the $\mathrm{SiC}$ feature appears along with an additional component peaking around 8.5-9.0 $\mu \mathrm{m}$ (class $\mathrm{SiC}+$ ). In a few stars the 8-9 $\mu \mathrm{m}$ feature rivals or exceeds the $\mathrm{SiC}$ feature in strength (class $\mathrm{SiC}++$ ).

The classic $\mathrm{SiC}$ class contains mostly Mira variables, while the $\mathrm{SiC}+$ and $\mathrm{SiC}++$ classes contain mostly semi-regular and irregular variables. The periods of the classic $\mathrm{SiC}$ sources are longer than those of the $\mathrm{SiC}+$ sources; that is true for both the Miras and the SRb variables. Classic SiC sources tend to have slightly redder [12]-[25] colors and correspondingly lower photospheric temperatures than the $\mathrm{SiC}+$ and $\mathrm{SiC}++$ sources. The classic $\mathrm{SiC}$ feature appears to be superimposed on a featureless continuum most likely due to amorphous carbon or graphitic material and is strongest for Miras. The $\mathrm{C} / \mathrm{O}$ ratio increases along the sequence $(\mathrm{SiC}) \rightarrow(\mathrm{SiC}+)$ $\rightarrow(\mathrm{SiC}++)$ from an average of $1.07(\mathrm{SiC})$ to $1.2(\mathrm{SiC}+)$ to $1.3(\mathrm{SiC}++)$. Assuming that $a: \mathrm{C}-\mathrm{H}$ is the carrier of the $8-9 \mu \mathrm{m}$ feature (Goebel et al. 1995), we propose that this feature will strengthen with increasing $\mathrm{C} / \mathrm{O}$ ratio. Support for this suggestion can be found in the increasing strength of the $\mathrm{C}_{2} \mathrm{H}_{2}+\mathrm{HCN}$ absorption feature seen in the $13-15 \mu \mathrm{m}$ region and in the spectrum of VX And which has the largest $\mathrm{C} / \mathrm{O}$ ratio (1.76) and the strongest contribution from the $8-9 \mu \mathrm{m}$ feature.

Our sample of 99 stars was selected by cross-referencing the IRAS Point Source Catalog and the General Catalogue of Variable Stars. Four sources show no dust emission, 8 have a weak, broad feature that is difficult to clas- 
sify, and 5 of these sources show emission from oxygen-rich dust, including two well-known silicate-carbon stars (BM Gem and V778 Cyg), and two $\mathrm{S}$ or SC stars which have been classified at various times as carbon stars (S Lyr and ST Cam). On the other hand, NP Pup appears to be related to the CS stars (Bidelman, private communication).

\section{The S Stars}

Pure S stars with $\mathrm{C} / \mathrm{O}$ close to unity show an emission feature peaking around $10.5 \mu \mathrm{m}$ which is subtly different from the $10 \mu \mathrm{m}$ amorphous silicate or the $11.2 \mu \mathrm{m} \mathrm{SiC}$ feature (Little-Marenin \& Little 1988). Neither Hron et al. (1997) nor Sloan \& Price (1995) were able to clearly distinguish this S-star feature from other emission features by their methods. A direct superposition of the spectral shapes of the various emission features is needed.

In general $\mathrm{S}$ stars tend to have lower mass-loss rates and higher gasto-dust ratios than $\mathrm{M}$ or $\mathrm{C}$ stars, implying less efficient dust formation in their circumstellar shells (Bieging \& Latter 1.994). Gas-to-dust ratios are estimated to be between 400 and 1000, at, least a factor of two higher than for carbon stars, and hence strong dust emission features are not seen or expected.

I have identified seven S stars listed in the catalogue of Chen et al. (1995) which are listed as having very strong silicate features at 10 and $18 \mu \mathrm{m}$ in their IRAS LRS spectra (LRS 25-29): IRAS 07197-1451 = TT $\mathrm{CMa} ; 11169-6111 ; 15347-5555 ; 16490-4618 ; 19545-1122=\mathrm{V} 1407$ Aql; $21029+4917$; and $22512+6100=$ V386 Cep. However, all seven stars are actually either M or MS stars rather than pure S stars (Little-Marenin 2000), and hence they reflect the mass-loss rates and dust content associated with M stars.

I suggest that among true $\mathrm{S}$ stars we should find a few stars with strong $10 \mu \mathrm{m}$ silicate features and enhanced ${ }^{13} \mathrm{C}$ content with ${ }^{12} \mathrm{C} /{ }^{13} \mathrm{C}$ ratios near the CNO equilibrium value of 3.4 rather than having values around 20, typical for S stars. If these stars are found they could be precursors to the Silicate-Carbon stars (Little-Marenin 1986), possibly produced by a helium core flash. However, no consensus about the evolutionary status of the Silicate-Carbon stars exists as yet. The MS stars with strong silicate features may fall into this category since all other MS stars I have analyzed have only weak silicate emission features. But no enhancement of ${ }^{13} \mathrm{C}$ has yet been identified (or searched for) in these stars.

The spectra of carbon-rich proto-planetary nebulae show an emission feature around $21 \mu \mathrm{m}$. 


\section{Conclusion}

Dust emission from circumstellar shells of AGB stars shows a variety of features in the $8 \cdots 22 \mu \mathrm{m}$ region. Besides the 10 and $18 \mu \mathrm{m}$ amorphous silicate features, $\mathrm{M}$ stars have additional emission at $11 \mu \mathrm{m}$ (crystalline olivine) and at $13.1 \mu \mathrm{m}$ (unknown carrier), as well as a broad, low-contrast feature $(9-15 \mu \mathrm{m})$ probably due to aluminum oxide. Most $\mathrm{C}$ stars show the classic $\mathrm{SiC}$ feature $(11.2 \mu \mathrm{m})$, but many also show an 8-9 $\mu \mathrm{m}$ emission feature that strengthens with increasing $\mathrm{C} / \mathrm{O}$ ratio. A featureless continuum (amorphous carbon or graphitic material) underlies the emission features. The S star feature peaking around $10.5 \mu \mathrm{m}$ is difficult to identify except by directly comparing its shape to other emission features. A number of AGB stars show no dust emission. It has been suggested that the $11.3 \mu \mathrm{m}$ feature superimposed on the amorphous silicate feature seen in some supergiants is due to PAHs rather than crystalline olivine. This finding is very interesting but needs further verification.

This work was supported by NASA grant NAG5-1667 and by Wellesley College.

\section{References}

Bieging, J. H. \& Latter, W.B. 1994, $A p J, 422,765$

Chen, P. S., Gao, H. \& Jorissen, A. 1995, A\&A Supp., 113, 51

Goebel, J. H., Cheeseman, P. \& Gerbault, F. 1995 ApJ, 449, 246

Hron, J., Aringer, B. \& Kerschbaum, F. 1997, $A \& A A, 322,280$

Little-Marenin, I. R. 1986, ApJ, 307, L15

Little-Marenin, I. R. 2000, in IAU Symp. 177: The Carbon Star Phenomenon, ed. R. F. Wing (Kluwer), p. 558

Little-Marenin, I. R. \& Little, S. J. 1988, ApJ, 333, 305

Little-Marenin, I. R. \& Little, S. J. 1990, AJ, 99, 1173 (LML90)

Little-Marenin, I. R., Ramsay, M. E., Stephenson, C. B., Little, S. J. \& Price, S. D. 1987, $A J, 93,663$.

Little-Marenin, I. R., Sloan, G. C. \& Price, S. D. 2000, in IAU Symp. 177: The Carbon Star Phenomenon, ed. R. F. Wing (Kluwer), p. 559

Sloan, G. C., LeVan, P. D. \& Little-Marenin, I. R. 1996, ApJ, 463, 310

Sloan, G. C. \& Price, S. D. 1995, ApJ, 451, 758

\section{Discussion}

Elitzur: Extracting spectral features by subtracting a single-temperature blackbody is a dangerous procedure. The correct analysis must involve a full radiative transfer calculation in which the dust opacity, including features, is an input. Detailed results obtained otherwise cannot be trusted.

Little-Marenin: I agree that the best procedure for analyzing the lowresolution spectra is a full radiative transfer calculation, but my simple subtraction of a blackbody allows one to identify different dust components. 
[Unknown]: Why would you expect less dust emission at maximum light than at minimum?

Little-Marenin: At higher luminosity and temperature, the condensation radius should move outward, evaporating grains, while at minimum the dust grain radius moves inward creating larger amounts of dust.

Ivezić: I would like to comment on the "discrepancy" between the behavior of the light curve and the strength of the $10 \mu \mathrm{m}$ silicate feature. It is true that the optical depth should be larger during minimum light, but the strength of the feature can both decrease and increase with optical depth. Therefore, as the luminosity of the star changes, the feature strength can either increase or decrease.

Little-Marenin: Good.

Speck: The shift of the $\mathrm{SiC}$ peak from 11.2 to $11.4 \mu \mathrm{m}$ was attributed to a change from $\alpha-\mathrm{SiC}$ to $\beta$-SiC. However, the $\beta$-SiC feature peaks at a shorter wavelength than $\alpha-\mathrm{SiC}$, so this is unlikely. On the other hand, impurities in $\alpha-\mathrm{SiC}$ shift the peak to longer wavelength. My own work on the $8.6-8.9 \mu \mathrm{m}$ feature suggests that there is no trend with optical depth. Maybe this suggests no evolutionary trend in the 8.6-8.9 $\mu \mathrm{m}$ feature.

Little-Marenin: Thank you for the information on $\alpha$ - and $\beta-\mathrm{SiC}$. We find trends in the strength of the $8.9 \mu \mathrm{m}$ feature with varying $\mathrm{C} / \mathrm{O}$, but like you, we find no correlation with optical depth.

van der Bliek: The IRAS LRS spectra were calibrated using the spectrum of $\alpha$ Tau. However, the LRS spectrum of $\alpha$ Tau turned out to have an SiO absorption feature $(7-10 \mu \mathrm{m})$, and this absorption then shows up as an "emission feature" in spectra without $\mathrm{SiO}$ absorption (Cohen et al. 1992, $A J, 104,2030)$. Did you correct for this?

Little-Marenin: I corrected all the LRS spectra à la Cohen et al. (1992) and rescaled the spectra to the $12 \mu \mathrm{m}$ PSC fluxes for that date. 